\title{
Identification of a Novel EXT Mutation in A Kindred With Hereditary Multiple Exostoses Using Whole- Exome Sequencing and Overview of Mutation Spectrum
}

\section{yanhan deng}

Department of Rheumatology and Immunology, Tongji Hospital, Huazhong University of Science and Technology, No. 1095 Jiefang Avenue, Wuhan, 430030

\section{yujian liu}

Division of Cardiology, Department of Internal Medicine, Tongji Hospital, Tongji Medical College, Huazhong University of Science and Technology, Wuhan, 430030

wei tu

Department of Rheumatology and Immunology, Tongji Hospital, Huazhong University of Science and Technology, No. 1095 Jiefang Avenue, Wuhan, 430030

liu yang ( $\square$ liuliutree@tjh.tjmu.edu.cn )

Reproductive Medicine Center, Tongji Hospital, Tongji Medical College, Huazhong University of Science and Technology, Wuhan, 430030

\section{Research Article}

Keywords: EXT1, novel heterozygous, splicing-site mutation, glycosyltrasferases, Hereditary Multiple Osteochondromas(HMO)

Posted Date: September 16th, 2021

DOl: https://doi.org/10.21203/rs.3.rs-770260/v1

License: (1) This work is licensed under a Creative Commons Attribution 4.0 International License. Read Full License 


\section{Abstract}

Background: Hereditary Multiple Osteochondromas(HMO) is a rare genetic musculoskeletal disorder characterized by multiple osteochondromas that form near to the growth plates of many bones. Loss-offunction mutations in EXT1 or EXT2 that encode glycosyltrasferases are the causal mutations for most HMO patients.

Methods: After collecting the family history and clinical information, we used Whole-Exome Sequencing to find the pathogenic mutations in one Chinese Hereditary Multiple Exostoses pedigree. Sanger sequencing and relevant online databases were used to validate the screened variants. Lollipop plots were drew to map the reported mutations from online databases (Multiple Osteochondroma Mutation Database and clinvar)on a linear protein domains by MutationMapper.

Results: A novel heterozygous splicing-site mutation in gene EXT1 (NM_000127:exon5:c.1417+1G>C区 chr8:118834703) was found in this pedigree and mutation spectrum of genes EXT1 and EXT2 were demonstrated.

Conclusions: Our results help this pedigree to identify the pathogenic variant and guide the prenatal diagnosis, also expand the mutation spectrum in Hereditary Multiple Osteochondromas.

\section{Introduction}

Hereditary multiple osteochondromas (HMO), also called hereditary multiple exostoses (HME), is a rare genetic disease, inherited in an autosomal dominant manner. Genes EXT1 (8q24.11) and EXT2(11p12) are the causal genes[1, 2], with a penetrance nearly to $100 \%$. Another gene designated EXT3 on chromosome 1 between D19S413 and D19S221 still needs further supported data.[3]

The prevalence of $\mathrm{HMO}$ in western countries is estimated around 0.4 to 1 in 50000 , peak in Guam 50 in $50000[4]$. The major symptoms of the $\mathrm{HMO}$ are multiple osteochondromas and related skeletal deformities. These bony exostoses could affect all metaphyses and diaphyses of bones, commonly found at knees, arms, forearm, thigh, pelvis and shoulder. Patients with HME show symptoms at a young age with a palpable mass near long bones, often are diagnosed before 12 years old. The number of the exostoses and functional deformity grow with the time. The exostoses burden per individual is about 31.5 with the mean age of 28.2. Patients with an EXT1 genotype were reported to have significantly greater number of exostoses compared to EXT2 genotype.[5]

Osteochondroma is usually a benign bone mass with a malignant degeneration rates about $0.38-7.0 \%$ toward chondrosarcoma. [6, 7] About 3/4 occurred between ages 20-40 and more than half at the pelvis and proximal femur. [7] Surgical excision of the tumor with free margin is suggested and local recurrence is lower than $2 \%$ if it was completely resected. Somatic mutation of EXT1 was the molecular basis of chondrosarcoma. Complications of HMO include local compression of adjacent tissues, joints deformity 
and restricted joint motion, discrepancies of limb-length, shortened stature and osteoarthrosis. Pain and joints dysfunction require surgical treatment to relieve symptoms.

Both EXT1 and EXT2 gene products are glycosyltransferases, which play a important role in the chain elongation step of heparan sulfate synthesis. HS binds with core proteins to produce heparin sulfate proteoglycans(HSPGs), which are abundant in both cell membrane and extracellular matrix and also function with vital signaling proteins, including bone morphogenetic protein (BMP), to regulating cell function.[8, 9] Animal studies showed that both Ext1+/- and Ext2+/- heterozygous knockout mice developed osteochondromas at a low penetrance.[10,11] In contrast, mice with stochastic inactivation of ext1 in some chondrocytes demonstrate mulpitle exotoses and bone deformity mimicking human HMO. $[12,13]$ And also compound heterozygous Ext1+/- Ext2+/- mice and Ext1-deficient mice displayed longbones-associated exotosese. [14, 15] In line with the observation of three-dimensional chondrogenic pellet model, osteochondromas formation arises via a "second hit" that would further lower heparan sulfate level.[16]

Considering the genetic causes and the disease burden of HME, genetic counseling and PGD/PGS ( Preimplantation Genetic Diagnosis/Screening ) is highly suggested. In this study, we collect family history, clinical data, radiological images of a four-generation Chinese HME kindred. Whole-Exome Sequencing was used to identify the pathogenic mutation in this family. Moreover, we review the published literature and two online Multiple Osteochondroma Mutation databases to figure out the hotspot and mutation sprectrum of EXT1 and EXT2.

\section{Material And Methods}

\section{Patients}

A four-generation Chinese kindred was studied with the permission of the family members, 11 out of 22 members were affected with HME ( 6 female, 5 male, 5 deceased).The proband III2, a 53 years old female, visited the rheumatologic department outpatient clinic for multiple joints pain. The palpable mass near the left knee was first noticed at the age 3 and gradually exostoses were affected both knee. She was born of nonconsaguineous Chinese parents with a family history. Physical examinations revealed exostoses in bilateral knee joints and left waist. The patient IV 3 was a 24 years old male with palpable lesions at the bilateral knees and elbow joints. The diagnosis was made by Multi disciplinary team of rheumatology, orthopedic and radiolagic department based on clinical manifestations and radiological images. The other family members' information was recorded due to statements of the proband and family photos.

\section{Genetic test}

We did Whole-Exome Sequencing of the proband III2, patient IV 3 on Illumina NovaSeq6000 according to the manufacturer's instruction(IIlumina, San Diego, CA, USA).Genomic DNA samples were extracted from 
peripheral blood using QIAamp RBlood Mini Kit (Qiagen, Hilden, Germany). The quality of genomic DNA was evaluated by agarose gel electrophoresis analysis, and the quantity was measured by

NanoDrop2000 and Qubit3.0. DNA was sheared with M220 Focused-ultrasonicator (Covaris, Woburn, MA, USA). DNA

target regions were captured by hybridizing the genomic DNA

sample library with the xGen RExome Research Panel v1.0 (IDT,

USA). The captured and amplified DNA samples were then sequenced

using Illumina NovaSeq6000 (Illumina, San Diego, CA, USA) with 150 base-paired end reads. The sequence reads were mapped and aligned to Hg37/GRP. Based on Standards and Guidelines for the Interpretation of Sequence Variants recommended by The American College of Medical Genetics and Genomics (ACMG), we analysed the sequencing data.[17]

The following databases were used to filter the variants, the 1000 Genomes Project (http://www.1000genomes.org), Exome Variant Server (http://evs.gs.washington.edu), and Exome Aggregation Consortium (http://exac.broadinstitute.org). Mutation Taster

(http://www.mutationtaster.org), PolyPhen-2 (http://genetics.bwh.harvard.edu/pph2 ), and SIFT (Sorting Intolerant from Tolerant) human protein (http://sift.jcvi.org/www/SIFT_enst_submit.html) software

programs were used was used to predict the destructive of amino acid substitution on structure and protein function. Three tools were used to

predict the splice-site variant, namely Human Splicing Finder (http://www.umd.be/HSF3/HSF.shtml), GeneSplicer (http://www.cbcb.umd.edu/software/GeneSplicer/gene_spl), NetGene2(http://www.cbs.dtu.dk/services/NetGene2/). Sanger Sequencing was tested to validate the variants.

To figure out the mutation spectrum of EXT1 and EXT2, we studied the mutations that reported in Multiple Osteochondroma Mutation Database(https://databases.lovd.nl/shared/genes) and Clinivar( https://www.ncbi.nlm.nih.gov/clinvar). The type of mutations was mapped on a linear protein and its function domain (Lollipop plot) by using the online MutationMapper (https://www.cbioportal.org/visualize) $[18,19]$. The type of variants was also studied.

\section{Resuts}

The pedigree of the HME kindred was showed in Figure IA, According to the clinical classification of Mordenti, these 2 patients were moderate type of IB class[20] . Genetic tests found that the proband was with a novel heterozygous splicing-site mutation in gene EXT1 (NM_000127:exon5:c.1417+1G>CD chr8:118834703). This mutation was further validated by Sanger sequencing (Figure IB) and also tested 
in affected and unaffected family members. Affected members had the same mutation and the unaffected ones were negative. The Radiological image of the proband was demonstrated in Figure (IC-D, CT scan and MRI respectively).

EXT1 and EXT2 are the two causal genes for Multiple Osteochondroma. according to the Multiple Osteochondroma Mutation Database (https://databases.lovd.nl/ , Date last updated August 18, 2021) [20] and Clinivar ( https://www.ncbi.nlm.nih.gov/clinvar), we found that no hotspot for EXT1, but for EXT2 the mutation variants were mainly located in the Exostosin region. (Figure2). Of all the 844 variants in EXT1 were reported, in them 714 were unique public pathogenic variants (49\% substitutions, 38\% deletions, $9 \%$ duplications in DNA level), $11 \%$ were located in splice region and $85 \%$ in coding region, and $52 \%$ were frameshifts and $28 \%$ were stop changes and $16 \%$ missense changes in protein level. For gene EXT2 totally 449 public DNA variants were reported, in them 362 variants were pathogenic (65\% substitutions, $26 \%$ deletions, $6 \%$ duplications in DNA level), and $79 \%$ located in coding region and $15 \%$ were in Splice region. In protein level 51\% were frameshifts and 29\% stop changes and 15\% missense changes.(details in supplements table1-2)

\section{Discussion}

This study indentified a novel splicing-site mutation in EXT1 from a four-generation HME Chinese family. All 2 affected ones which we did genetic tests were found the same site and the unaffected members were not. For both EXT1 and EXT2, the most common mutation type is substitution, followed by deletion and duplication, located mainly on coding region and more than $10 \%$ in splicing region. And unlike EXT1, mutation sites of EXT2 were focused on the exostosin region,and rarely in the glycol-transf-64 domain. Consistent with the previous study suggested that mutation hot-spot region of EXT2 were Exon 5. [21]

One limitation of this study is that we did not test all the affected patients, this was due to geographic distance and personal will. Both patients complained about pain and shyness in school. A national wide study in the Netherlands showed that multiple hereditary exostoses damaged both physical and psychological well-being. Pain was the major complain, and about half school attendees experienced problems at school and more than a quarter were unable to join the sport activities.[22] HME impact on patients quality of life similar to osteoarthritis in the mental aspect and women responded even worse in the psychological side. [23]

Another limitation is that the diagnosis was based on the family history, clinical manifestations and radiological results, pathologic diagnosis was lacked because family members were moderate type of IB and did not do the surgical removement. Up to now no consensus on the hereditary multiple osteochondromas (HMO) diagnosis criteria was reported. And clinically

The diagnosis was made when patients with more than one osteochondromas of the juxta-epiphyseal region of long bones was confirmed by radiological images. Additionally these patients were with a positive family history and/or genetic tests indicated positive in gene EXT1 and EXT2. [24] Differential diagnosis was made from Dysplasia Epiphysealis Hemimelica, metachondromatosis (MC), 
enchondromatosis (Ollier disease and Maffucci syndrome). And the female patient was short-stature, while the other patients were within normal range. Diminished stature was observed in about $37 \%-53 \%$ patients and more severely affected in patients with an EXT1 mutation.[4,25] The affected patients in this studied showed no sign of malignant degeneration. A international, web-based survey showed that about $2.7 \%$ patients underwent malignant transformation at a mean age of 28.6 years. This occurred most commonly on pelvis (8/21)and scapula $(4 / 21)$, followed by proximal femur, rib, skull base, tibia and foot.[6] Whether genetic variants plays a role in the malignant transformation risk is undetermined yet. The molecular basis and characteristic of the HMO patients who had malignant change to chondrosarcoma still need further investigations. A regular doctor visit and yearly physical examination is strongly recommended for the malignant risk.

In conclusion, we found a novel splice-site variant in EXT1 in one Chinese hereditary multiple osteochondromas family. Additionally by digging the online

Multiple Osteochondroma Mutation Database and clinvar, we present the mutation spectrum and lollipop of variants site on protein domains. Multiple Osteochondromas is a chronic skeletal disease with a certain risk of malignant transformation. Medical managements, includes early diagnosis, genetic counseling, regular medical follow-up help to improve the patients' clinical outcomes. Joint effort of social support and school care are required to help the $\mathrm{HMO}$ patients to maintain physical and mental well-being. More cohorts and pathogenesis investigations are needed elucidate the correlation of the genetic basis and clinical manifestations.

\section{Abbreviations}

HMO Hereditary Multiple Osteochondromas

HME Hereditary Multiple Exostoses

EXT1 Exostosin Glycosyltransferase 1

EXT1 Exostosin Glycosyltransferase 2

\section{Declarations}

Ethics approval and consent to participate: This study was approved by the Ethics Committee of Tongji Hospital and informed written consent was obtained from all the participants and/or their legal guardian(s) for deceased participants. All the methods were carried out in accordance with relevant guidelines and regulations.

Consent for publication: Not Applicable

Availability of Data and Materials: The datasets generated during the current study are available in the following website link 
Competing interests: The authors declare that they have no competing interests

Funding: This research was supported by grant (Nr1210487149) from the Research Fund of Tongji hospital.

Authors' contributions: WT and YL organized this study and interpreted the patient data. YJL and YHD analysed the data. YHD was a major contributor in writing the manuscript. All authors read and approved the final manuscript.

Acknowledgements: Thanks to Wenjie zhu from Radiology Department Tongji Hospital for helps in CT and MRI images. We show gratitude to the HMO family members who participated in this study.

\section{References}

1. Ahn J, Ludecke HJ, Lindow S, Horton WA, Lee B, Wagner MJ, Horsthemke B, Wells DE: Cloning of the putative tumour suppressor gene for hereditary multiple exostoses (EXT1). Nat Genet 1995, 11(2):137-143.

2. Stickens D, Clines G, Burbee D, Ramos P, Thomas S, Hogue D, Hecht JT, Lovett M, Evans GA: The EXT2 multiple exostoses gene defines a family of putative tumour suppressor genes. Nat Genet 1996, 14(1):25-32.

3. Le Merrer M, Legeai-Mallet L, Jeannin PM, Horsthemke B, Schinzel A, Plauchu H, Toutain A, Achard F, Munnich A, Maroteaux P: A gene for hereditary multiple exostoses maps to chromosome 19p. Hum Mol Genet 1994, 3(5):717-722.

4. Schmale GA, Conrad EU, 3rd, Raskind WH: The natural history of hereditary multiple exostoses. $J$ Bone Joint Surg Am 1994, 76(7):986-992.

5. Clement ND, Porter DE: Hereditary multiple exostoses: anatomical distribution and burden of exostoses is dependent upon genotype and gender. Scott Med J 2014, 59(1):35-44.

6. Czajka CM, DiCaprio MR: What is the Proportion of Patients With Multiple Hereditary Exostoses Who Undergo Malignant Degeneration? Clin Orthop Relat Res 2015, 473(7):2355-2361.

7. Fei L, Ngoh C, Porter DE: Chondrosarcoma transformation in hereditary multiple exostoses: $A$ systematic review and clinical and cost-effectiveness of a proposed screening model. J Bone Oncol 2018, 13:114-122.

8. Bishop JR, Schuksz M, Esko JD: Heparan sulphate proteoglycans fine-tune mammalian physiology. Nature 2007, 446(7139):1030-1037.

9. Mundy C, Yang E, Takano H, Billings PC, Pacifici M: Heparan sulfate antagonism alters bone morphogenetic protein signaling and receptor dynamics, suggesting a mechanism in hereditary multiple exostoses. J Biol Chem 2018, 293(20):7703-7716. 
10. Stickens D, Zak BM, Rougier N, Esko JD, Werb Z: Mice deficient in Ext2 lack heparan sulfate and develop exostoses. Development 2005, 132(22):5055-5068.

11. Hilton MJ, Gutierrez L, Martinez DA, Wells DE: EXT1 regulates chondrocyte proliferation and differentiation during endochondral bone development. Bone 2005, 36(3):379-386.

12. Matsumoto K, Irie F, Mackem S, Yamaguchi Y: A mouse model of chondrocyte-specific somatic mutation reveals a role for Ext1 loss of heterozygosity in multiple hereditary exostoses. Proc Nat/ Acad Sci U S A 2010, 107(24):10932-10937.

13. Jones KB, Piombo V, Searby C, Kurriger G, Yang B, Grabellus F, Roughley PJ, Morcuende JA, Buckwalter JA, Capecchi MR et al: A mouse model of osteochondromagenesis from clonal inactivation of Ext1 in chondrocytes. Proc Natl Acad Sci U S A 2010, 107(5):2054-2059.

14. Sgariglia F, Candela ME, Huegel J, Jacenko O, Koyama E, Yamaguchi Y, Pacifici M, Enomoto-Iwamoto M: Epiphyseal abnormalities, trabecular bone loss and articular chondrocyte hypertrophy develop in the long bones of postnatal Ext1-deficient mice. Bone 2013, 57(1):220-231.

15. Zak BM, Schuksz M, Koyama E, Mundy C, Wells DE, Yamaguchi Y, Pacifici M, Esko JD: Compound heterozygous loss of Ext1 and Ext2 is sufficient for formation of multiple exostoses in mouse ribs and long bones. Bone 2011, 48(5):979-987.

16. Reijnders CM, Waaijer CJ, Hamilton A, Buddingh EP, Dijkstra SP, Ham J, Bakker E, Szuhai K, Karperien M, Hogendoorn PC et al: No haploinsufficiency but loss of heterozygosity for EXT in multiple osteochondromas. Am J Pathol 2010, 177(4):1946-1957.

17. Richards S, Aziz N, Bale S, Bick D, Das S, Gastier-Foster J, Grody WW, Hegde M, Lyon E, Spector E et al: Standards and guidelines for the interpretation of sequence variants: a joint consensus recommendation of the American College of Medical Genetics and Genomics and the Association for Molecular Pathology. Genet Med 2015, 17(5):405-424.

18. Gao J, Aksoy BA, Dogrusoz U, Dresdner G, Gross B, Sumer SO, Sun Y, Jacobsen A, Sinha R, Larsson E et al: Integrative analysis of complex cancer genomics and clinical profiles using the cBioPortal. Sci Signal 2013, 6(269):pl1.

19. Cerami E, Gao J, Dogrusoz U, Gross BE, Sumer SO, Aksoy BA, Jacobsen A, Byrne CJ, Heuer ML, Larsson $\mathrm{E}$ et al: The cBio cancer genomics portal: an open platform for exploring multidimensional cancer genomics data. Cancer Discov 2012, 2(5):401-404.

20. Mordenti M, Ferrari E, Pedrini E, Fabbri N, Campanacci L, Muselli M, Sangiorgi L: Validation of a new multiple osteochondromas classification through Switching Neural Networks. Am J Med Genet $A$ 2013, 161A(3):556-560.

21. Yang A, Kim J, Jang JH, Lee C, Lee JE, Cho SY, Jin DK: Identification of a novel mutation in EXT2 in a fourth-generation Korean family with multiple osteochondromas and overview of mutation spectrum. Ann Hum Genet 2019, 83(3):160-170.

22. Goud AL, de Lange J, Scholtes VA, Bulstra SK, Ham SJ: Pain, physical and social functioning, and quality of life in individuals with multiple hereditary exostoses in The Netherlands: a national cohort study. J Bone Joint Surg Am 2012, 94(11):1013-1020. 
23. D'Ambrosi R, Ragone V, Caldarini C, Serra N, Usuelli FG, Facchini RM: The impact of hereditary multiple exostoses on quality of life, satisfaction, global health status, and pain. Arch Orthop Trauma Surg 2017, 137(2):209-215.

24. Bovee JV: Multiple osteochondromas. Orphanet J Rare Dis 2008, 3:3.

25. Clement ND, Duckworth AD, Baker AD, Porter DE: Skeletal growth patterns in hereditary multiple exostoses: a natural history. J Pediatr Orthop B 2012, 21(2):150-154.

\section{Figures}

A

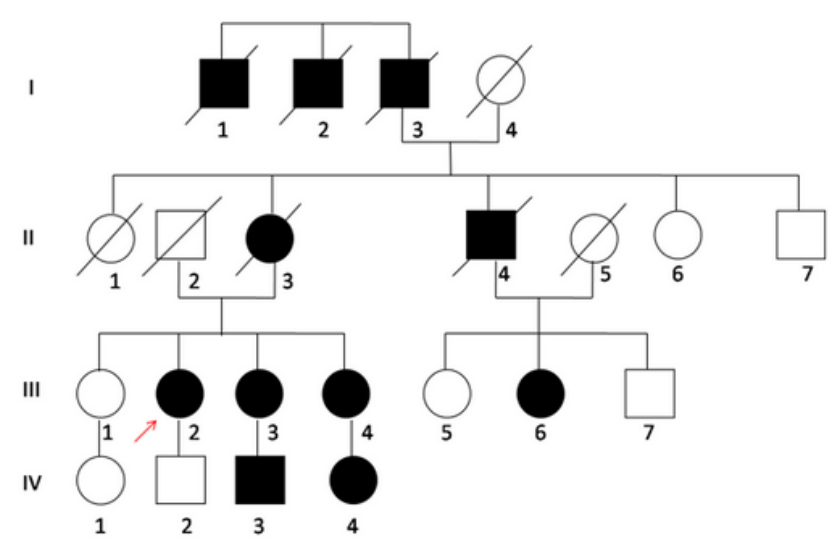

c

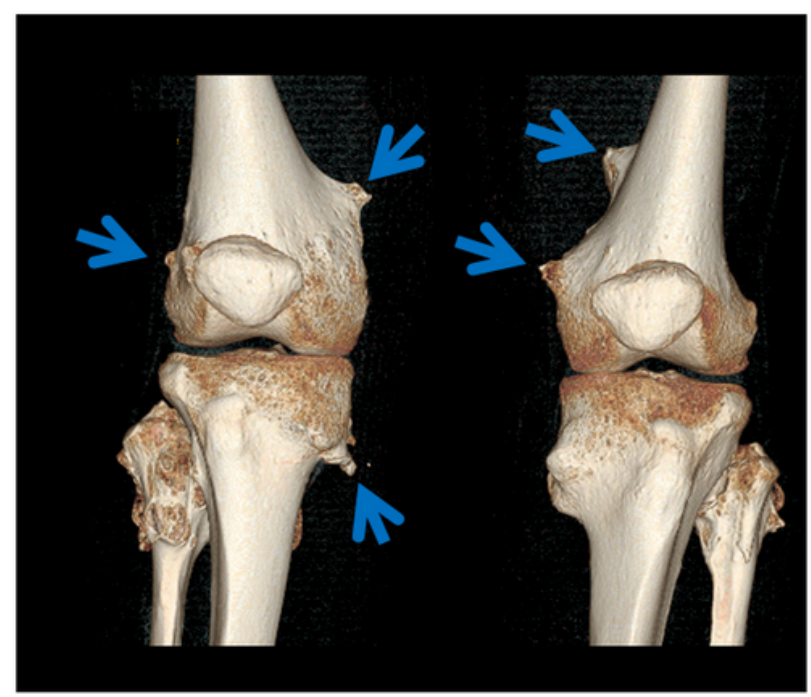

B

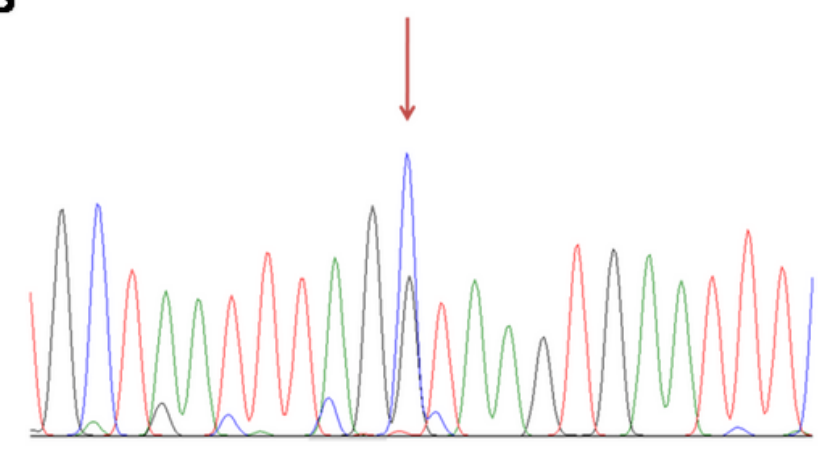

NM_000127.3(EXT1):c.1417+1G>C

D

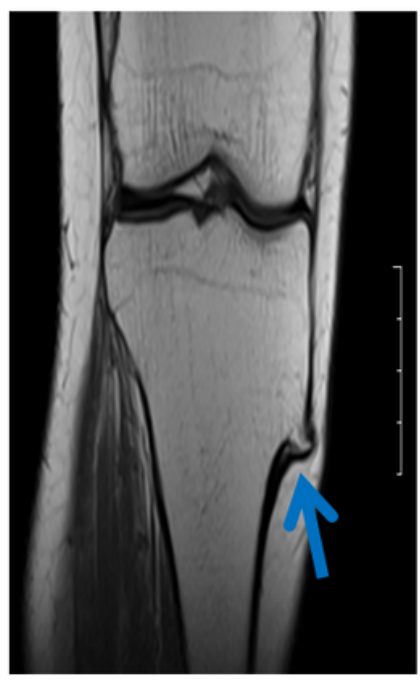

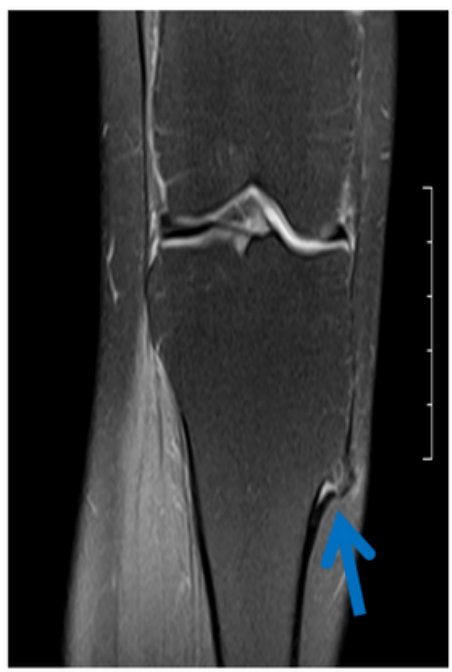

\section{Figure 1}

(a) Family pedigree of the HMO with EXT1 gene mutation. Generation was demonstrated as I to IV. Patient III-2 is the proband. Black and white indicate the family member affected or unaffected respectively by HMO. (b) Whole-exome-sequencing found a novel EXT1 frameshift mutation(NM_000127:exon5:c.1417+1G>C『chr8:118834703) in this pedigree. Sanger sequencing shows a heterozygous deletion in proband. (c) CT scan of proband's knee, exostoses are denoted by arrow . Both 
distal femur and right proximal tibie are affected. (d) T1-weighted and T2-weighted MRI scan of proband's left proximal tibia. Arrow indicated the cartilage cap.

\section{A}

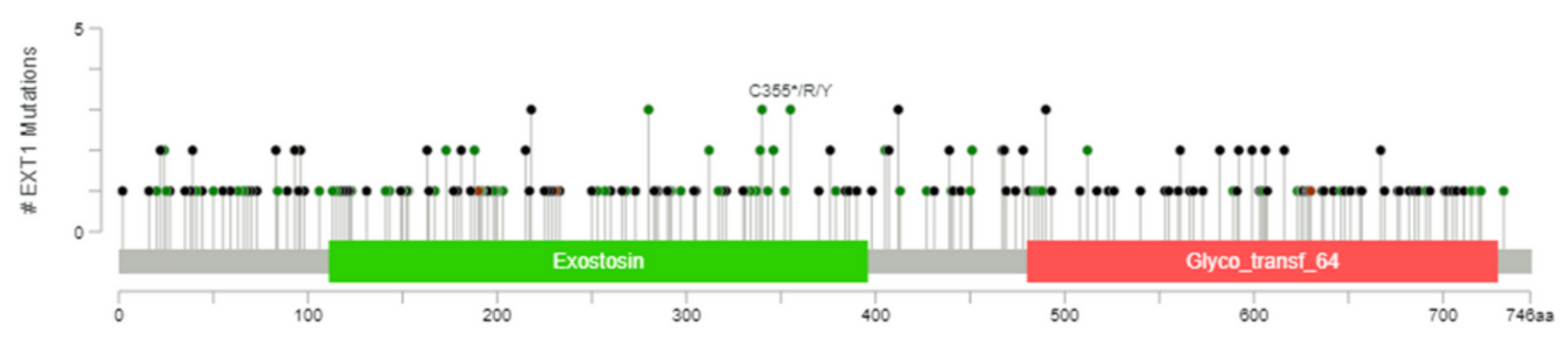

B

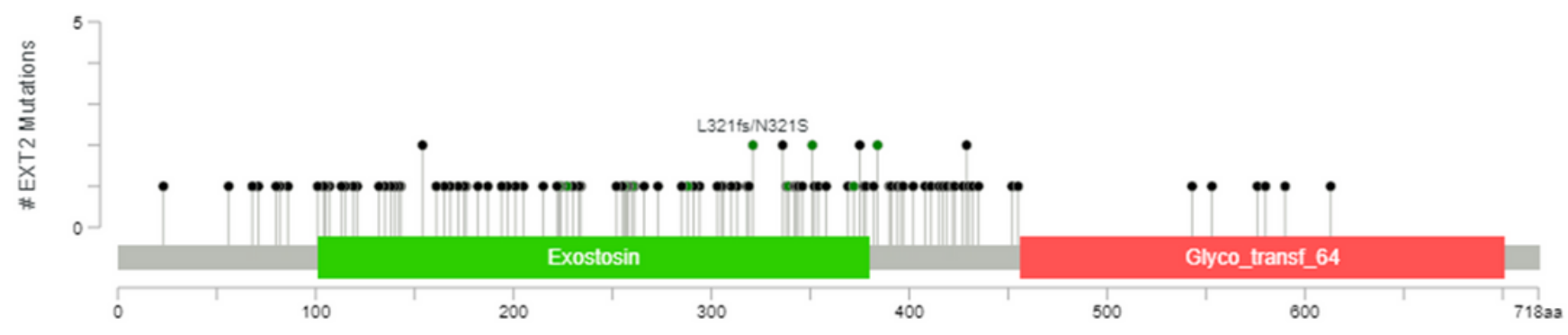

Figure 2

(A)(B) Mutations spectrum on a linear protein domain of EXT1 and EXT2 were demonstrated respectively. Missense variants (green), Truncating variants (black), Inframe variants (brown) was labeled

\section{Supplementary Files}

This is a list of supplementary files associated with this preprint. Click to download.

- SupplementTable1EXT1.pdf

- SupplementTable2EXT2.pdf 